\title{
Breath-synchronized electrical stimulation of the expiratory muscles in mechanically ventilated patients: a randomized controlled feasibility study and pooled analysis
}

Annemijn H. Jonkman 1,2 ${ }^{10}$, Tim Frenzel ${ }^{3}$, Euan J. McCaughey ${ }^{4,5}$, Angus J. McLachlan ${ }^{6}$, Claire L. Boswell-Ruys ${ }^{4,5}$, David W. Collins ${ }^{7}$, Simon C. Gandevia ${ }^{4,5}$, Armand R. J. Girbes ${ }^{1,2}$, Oscar Hoiting ${ }^{8}$, Matthijs Kox ${ }^{3}$, Eline Oppersma ${ }^{9}$, Marco Peters ${ }^{8}$, Peter Pickkers ${ }^{3}$, Lisanne H. Roesthuis ${ }^{3}$, Jeroen Schouten ${ }^{3}$, Zhong-Hua Shi ${ }^{1,2}$, Peter H. Veltink ${ }^{10}$, Heder J. de Vries ${ }^{1,2}$, Cyndi Shannon Weickertt ${ }^{41,12}$, Carsten Wiedenbach ${ }^{8}$, Yingrui Zhang ${ }^{1}$, Pieter R. Tuinman ${ }^{1,2}$, Angélique M. E. de Man ${ }^{1,2}$, Jane E. Butler ${ }^{4,5}$ and Leo M. A. Heunks ${ }^{1,2^{*}}$

\begin{abstract}
Background: Expiratory muscle weakness leads to difficult ventilator weaning. Maintaining their activity with functional electrical stimulation (FES) may improve outcome. We studied feasibility of breath-synchronized expiratory population muscle FES in a mixed ICU population ("Holland study") and pooled data with our previous work ("Australian study") to estimate potential clinical effects in a larger group.

Methods: Holland: Patients with a contractile response to FES received active or sham expiratory muscle FES (30 min, twice daily, 5 days/week until weaned). Main endpoints were feasibility (e.g., patient recruitment, treatment compliance, stimulation intensity) and safety. Pooled: Data on respiratory muscle thickness and ventilation duration from the Holland and Australian studies were combined $(N=40)$ in order to estimate potential effect size. Plasma cytokines (day 0,3 ) were analyzed to study the effects of FES on systemic inflammation.

Results: Holland: A total of 272 sessions were performed (active/sham: 169/103) in 20 patients ( $N=$ active/sham: 10/10) with a total treatment compliance rate of $91.1 \%$. No FES-related serious adverse events were reported. Pooled: On day 3, there was a between-group difference ( $N=$ active/sham: $7 / 12)$ in total abdominal expiratory muscle thickness favoring the active group [treatment difference (95\% confidence interval); $2.25(0.34,4.16) \mathrm{mm}, P=0.02$ ] but not on day 5. Plasma cytokine levels indicated that early FES did not induce systemic inflammation. Using a survival analysis approach for the total study population, median ventilation duration and ICU length of stay were 10 versus 52 $(P=0.07)$, and 12 versus $54(P=0.03)$ days for the active versus sham group. Median ventilation duration of patients that were successfully extubated was $8.5[5.6-12.2]$ versus $10.5[5.3-25.6]$ days $(P=0.60)$ for the active $(N=16)$ versus sham $(N=10)$ group, and median ICU length of stay was 10.5 [8.0-14.5] versus 14.0 [9.0-19.5] days $(P=0.36)$ for those
\end{abstract}

\footnotetext{
*Correspondence: L.Heunks@amsterdamumc.nl

${ }^{1}$ Department of Intensive Care Medicine, Amsterdam University Medical

Centers, location VUmc, Postbox 7505, 1007 MB Amsterdam, The

Netherlands

Full list of author information is available at the end of the article
}

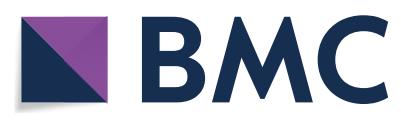

(c) The Author(s) 2020. Open Access This article is licensed under a Creative Commons Attribution 4.0 International License, which permits use, sharing, adaptation, distribution and reproduction in any medium or format, as long as you give appropriate credit to the original author(s) and the source, provide a link to the Creative Commons licence, and indicate if changes were made. The images or other third party material in this article are included in the article's Creative Commons licence, unless indicated otherwise in a credit line to the material. If material is not included in the article's Creative Commons licence and your intended use is not permitted by statutory regulation or exceeds the permitted use, you will need to obtain permission directly from the copyright holder. To view a copy of this licence, visit http://creativecommons.org/licenses/by/4.0/. The Creative Commons Public Domain Dedication waiver (http://creativeco mmons.org/publicdomain/zero/1.0/) applies to the data made available in this article, unless otherwise stated in a credit line to the data. 
active $(N=16)$ versus sham $(N=8)$ patients that were extubated and discharged alive from the ICU. During ICU stay, $3 / 20$ patients died in the active group versus $8 / 20$ in the sham group $(P=0.16)$.

Conclusion: Expiratory muscle FES is feasible in selected ICU patients and might be a promising technique within a respiratory muscle-protective ventilation strategy. The next step is to study the effects on weaning and ventilator liberation outcome.

Trial registration: ClinicalTrials.gov, ID NCT03453944. Registered 05 March 2018—Retrospectively registered, https:// clinicaltrials.gov/ct2/show/NCT03453944.

Keywords: Functional electrical stimulation, Expiratory muscles, Mechanical ventilation

\section{Background}

The respiratory muscle pump drives alveolar ventilation. The diaphragm is the most prominent inspiratory muscle, while abdominal wall muscles play an important role in active expiration $[1,2]$. Respiratory muscle weakness is highly prevalent in critically ill intensive care unit (ICU) patients and associated with difficult ventilator weaning, prolonged ICU stay, and mortality [3-10]. Mechanical ventilation is a critical contributor as excessive ventilator assist may result in diaphragm disuse atrophy $[3,4,11]$. Indeed, maintaining diaphragm activity during mechanical ventilation may preserve diaphragm function [4]. Moreover, in a preclinical study, electrical stimulation of the phrenic nerves was shown to attenuate diaphragm atrophy resulting from controlled mechanical ventilation [12].

In contrast, the impact of mechanical ventilation on the expiratory muscles is less well studied and interventions targeting these muscles are lacking. This is surprising, as the expiratory muscles are vital for airway clearance, prevention of atelectasis, and enhancement of minute ventilation in conditions of low inspiratory muscle capacity and/or high respiratory load [1]. Development of expiratory muscle weakness during ICU stay has been associated with extubation failure and (re)hospitalization due to respiratory complications $[9,10,13,14]$. Maintaining their activity under mechanical ventilation is therefore likely to improve outcome.

Functional electrical stimulation (FES) of limb muscles has been studied extensively as a strategy to limit disuse atrophy and to improve muscle function during prolonged immobilization $[15,16]$. FES uses electrical currents to generate artificial skeletal muscle contraction without patient cooperation, making it an attractive intervention in uncooperative mechanically ventilated patients. Recently, we published results of a pilot study employing noninvasive breath-synchronized FES of the expiratory abdominal wall muscles, further referred to as 'expiratory muscle FES', in 20 ICU patients ("Australian study") [17] and showed that this intervention has potential benefits. However, the active group of this single-center study consisted mainly of neurocritically ill patients $(70 \%)$ and results need to be confirmed in a more diverse ICU population. The aim of the current study ("Holland study") was to assess feasibility of performing expiratory muscle FES in a mixed ICU population. Additionally, data from both studies were pooled to estimate potential clinical effects in a larger, more heterogeneous patient group.

\section{Methods}

See Additional file 1 for additional details.

\section{Holland study}

\section{Participants and Study design}

Twenty patients were randomized in this prospective sham-controlled feasibility study conducted in three mixed ICUs in the Netherlands (Radboud University Medical Center; Amsterdam UMC, location VUmc; Canisius Wilhelmina Ziekenhuis). Patients were enrolled as early as possible but within $72 \mathrm{~h}$ after intubation. Exclusion criteria were an anticipated stay on the ventilator $<72 \mathrm{~h}$ at the time of study enrolment, congenital myopathies or neuropathies, and contraindications for expiratory muscle FES (cardiac pacemaker, refractory epilepsy, recent $(<4$ weeks) abdominal surgery, body mass index $>35 \mathrm{~kg} / \mathrm{m}^{2}$, and pregnancy). Written informed consent was obtained from the patient's substitute decision-maker. Only patients with an adequate contractile response to expiratory muscle FES using stimulation settings similar as for the active group (see below and Additional file 1) were randomized.

\section{Expiratory muscle FES}

Expiratory muscle FES was applied for $30 \mathrm{~min}$, twice daily, for 5 days per week (first 5 days consecutively), until patients were weaned from mechanical ventilation, but no longer than six weeks. Local protocols for mechanical ventilation and weaning were followed.

Stimulation was applied during exhalation using an investigational device (VentFree model VK03-K, Liberate Medical LLC, USA) (Additional file 1: Figure 1 and [17]) via surface electrodes on the abdominal wall, and stimulation intensity was titrated in order to activate the external 
oblique, internal oblique and transversus abdominis muscles (Additional file 1: Figure 2). The patient's tolerance of expiratory muscle FES was continuously monitored by means of clinical judgment; sessions were discontinued in the presence of stop criteria (see Additional file 1). In the active group $(N=10)$, settings were as follows: frequency $30 \mathrm{~Hz}$, pulse width $352 \mu$ s and a current amplitude (intensity) set to cause strong muscle contraction, with a maximum intensity initially set at $60 \mathrm{~mA}$ (tolerated intensity in healthy volunteers [18]). After the first four patients were enrolled in the study, the protocol was amended to allow a maximum intensity of $100 \mathrm{~mA}$ (maximum device output). Strong muscle contraction was verified (visible and palpable) every ten minutes throughout each FES session, and if necessary, stimulation intensity was increased. In the sham group $(N=10)$, the following settings were applied and would allow a patient to have a sensation of stimulation without muscle contraction (as verified clinically and with ultrasound): frequency $10 \mathrm{~Hz}$, pulse width $352 \mu \mathrm{s}$, intensity $10 \mathrm{~mA}$.

\section{Data collection}

During each stimulation session, ventilator parameters and vital signs were collected. Ultrasound measurements were performed (see Additional file 1 and [19]) to study changes in respiratory muscle thickness (external oblique, internal oblique and transversus abdominis (their combined thickness is further referred to as total abdominal expiratory muscle thickness), rectus abdominis thickness and diaphragm thickness). The researcher administering expiratory muscle FES did not perform ultrasound recordings, and outcome assessors were not in the patient room when stimulation was delivered. Blood samples for the analysis of plasma cytokine levels were collected at baseline and on day 3 (see Additional file 1). Ventilation duration was collected as the number of days from mechanical ventilation onset until the first successful extubation [i.e., no need for ventilator support (no noninvasive ventilation and no high-flow nasal therapy) for $48 \mathrm{~h}$ after extubation].

\section{Outcomes}

The primary outcome was the feasibility of performing expiratory muscle FES in terms of patient recruitment, contractile response, and treatment compliance, and safety. As an additional safety endpoint, plasma cytokine levels were studied to evaluate whether early application of FES was associated with enhanced systemic proinflammatory response. This was evaluated for the full analysis set (per-protocol analysis). Ultrasound and clinical data of the Holland study were evaluated in a pooled analysis.

\section{Pooled analysis}

Data from the Holland and Australian studies could be combined $(N=40)$ as FES protocols were similar until extubation. Pooled endpoints were respiratory muscle thickness, plasma cytokine levels, ventilation duration, ICU length of stay, and ICU mortality. Ultrasound data were combined for patients with a treatment compliance of $\geq 75 \%$ treatment days from the intent-to-treat set and until day 5 after enrollment, as ultrasound protocols were similar until this point. Other pooled endpoints were analyzed for the full analysis set (per-protocol analysis).

\section{Statistics}

Data are presented as medians with interquartile range $\left[q_{1}-q_{3}\right]$, mean \pm standard deviation, or percentages. Statistical analyses were performed using two-sided hypothesis tests at the $5 \%$ significance level.

\section{Holland study}

As this is a feasibility study and no data were available on the effects of expiratory muscle FES on expiratory muscle thickness or function when designing the study, we planned to enroll a convenience sample of ten subjects per group, 20 in total. Compliance to expiratory muscle FES sessions was calculated as the percentage of all sessions that should have been completed between randomization and study completion or withdrawal and was considered a continuous variable. Stimulation intensities and safety endpoints (adverse events) are presented using descriptive statistics. Changes in plasma cytokine levels were assessed using a two-way ANOVA with factors of group (active, sham) and time (measurements at baseline and on day 3). Further details are presented in Additional file 1.

\section{Pooled analysis}

Survival analysis was performed to estimate the median ventilation duration and ICU length of stay, applying the cumulative incidence competing risks method. Competing risks were death or withdrawal of treatment (e.g., ventilator support) with the intention of subsequent death. Participants who were alive but who did not experience the event of interest were right censored at the last available study day. Gray's test was used for comparison of cumulative incidence functions, and the median duration was defined as the time point where $50 \%$ of participants experienced the event of interest. In addition, we calculated the median $\left[q_{1}-q_{3}\right]$ ventilation duration and ICU length of stay for those patients that experienced the event of interest during the study period. Differences between these groups were assessed with the MannWhitney $U$ test, according to the distribution. Changes 
from baseline of total abdominal expiratory muscle thickness were analyzed with a linear mixed effects model with fixed factors of baseline thickness, group, assessment session and group by assessment session interaction, and a random effect of participant. For further statistical details and analyses of other outcome parameters, see Additional file 1 .

\section{Results}

Holland study Study population

Figure 1 presents the CONSORT flow diagram. Five patients did not show a contractile response to expiratory muscle FES $(N=2$ at $60 \mathrm{~mA}$ and $N=3$ at $100 \mathrm{~mA})$ and were withdrawn before randomization. No potential explanatory factors hampering contractile response could be identified in these patients (i.e., no recent neuromuscular blockers, no relevant medical history, and body mass index, sedation, and fluid status were not different from other patients). Baseline characteristics of the randomized patients ( $N=$ active/sham: 10/10) are presented in Table 1.

\section{Feasibility}

Table 2 presents expiratory muscle FES session compliance and adverse events. Nonserious adverse events categorized as 'possibly' or 'definitely' related to the intervention were reported in sixteen sessions ( $n=$ active/ sham sessions: $13 / 3$, percentage of total sessions per group active/sham: $7.7 \% / 2.9 \%$ ) in a total of five active patients and in one sham patient. In the active group, these events included discomfort $(n=3$ sessions, in two patients) and brief, spontaneous reversible episodes of hypertension and/or tachycardia ( $n=10$ sessions, in four

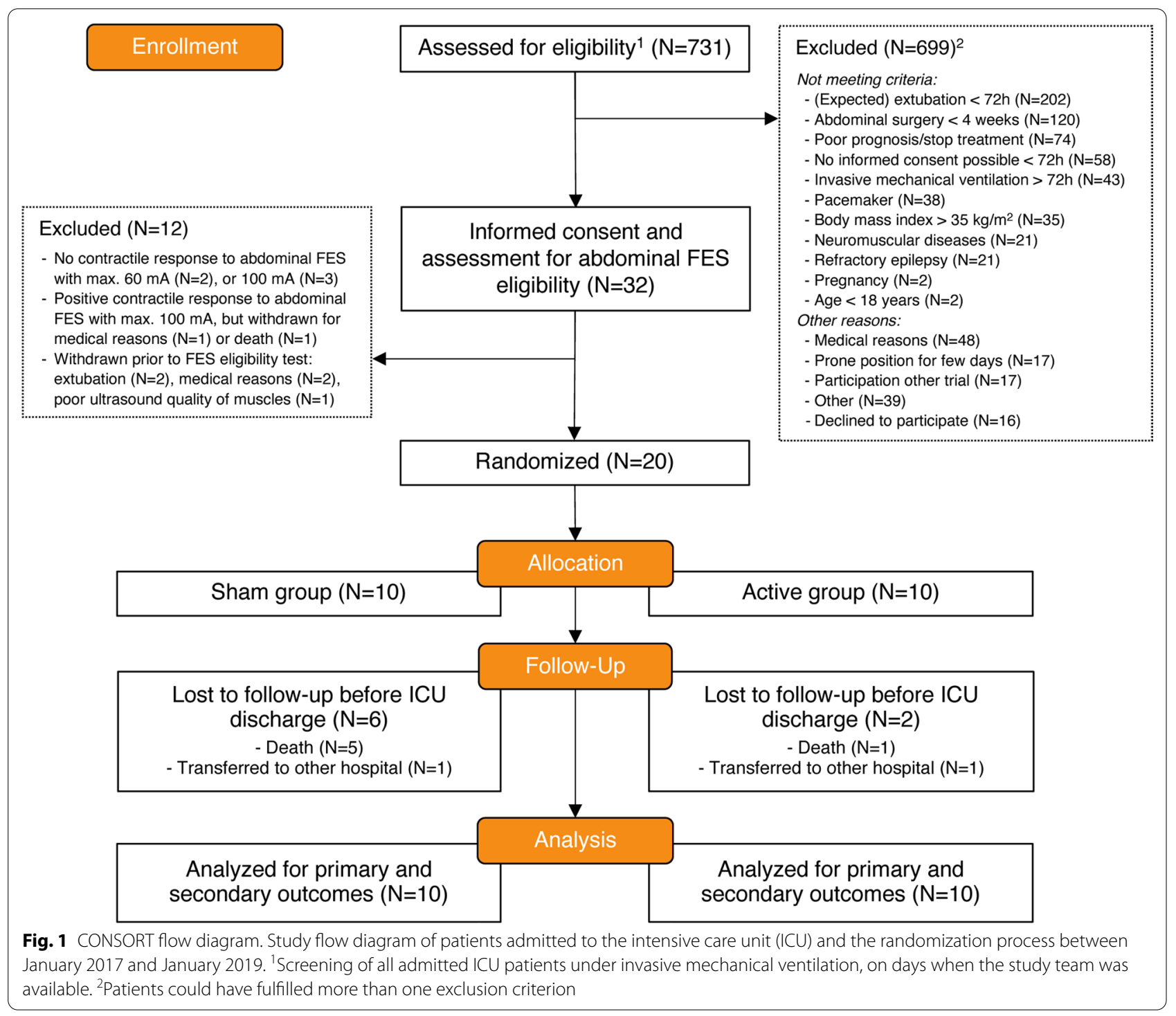


Table 1 Patient characteristics for the Holland study and the pooled data (including the Holland and Australian study)

\begin{tabular}{|c|c|c|c|c|c|c|}
\hline & \multicolumn{3}{|c|}{ Holland study } & \multicolumn{3}{|c|}{ Pooled data (Holland + Australia) } \\
\hline & All $(N=20)$ & Active $(N=10)$ & Sham $(N=10)$ & All $(N=40)$ & Active $(N=20)$ & Sham $(N=20)$ \\
\hline Age, years & $69.0 \pm 8.5$ & $72.2 \pm 6.1$ & $65.7 \pm 9.6$ & $63.4 \pm 14$ & $64.5 \pm 14.9$ & $62.4 \pm 13.3$ \\
\hline Sex, male/female & $14 / 6$ & $8 / 2$ & $6 / 4$ & $26 / 14$ & $15 / 5$ & $11 / 9$ \\
\hline Body mass index, $\mathrm{kg} / \mathrm{m}^{2}$ & $26.4 \pm 4.4$ & $25.9 \pm 5.1$ & $26.8 \pm 3.8$ & $26.4 \pm 4.4$ & $26.8 \pm 4.5$ & $26.5 \pm 3.2$ \\
\hline History of COPD, \% $(n / N)$ & $50(10 / 20)$ & $60(6 / 10)$ & $40(4 / 10)$ & $25(10 / 40)$ & $30(6 / 20)$ & $20(4 / 20)$ \\
\hline MV duration at enrollment, days & $2.5 \pm 1.0$ & $2.4 \pm 1.0$ & $2.5 \pm 1.0$ & $2.5 \pm 0.8$ & $2.5 \pm 0.9$ & $2.5 \pm 0.8$ \\
\hline $\mathrm{FiO}_{2}$ at study enrollment & $0.42 \pm 0.12$ & $0.42 \pm 0.09$ & $0.42 \pm 0.15$ & $0.36 \pm 0.13$ & $0.34 \pm 0.11$ & $0.37 \pm 0.15$ \\
\hline \multicolumn{7}{|l|}{ Primary reason for $\mathrm{MV}, \%(n / N)$} \\
\hline Cardiac arrest & $5(1 / 20)$ & $0(0 / 0)$ & $10(1 / 10)$ & $2.5(1 / 40)$ & $0(0 / 20)$ & $5(1 / 20)$ \\
\hline Pneumonia & $50(10 / 20)$ & $40(4 / 10)$ & $60(6 / 10)$ & $30(12 / 40)$ & $25(5 / 20)$ & $35(7 / 20)$ \\
\hline Postoperative & $5(1 / 20)$ & $10(1 / 10)$ & $0(0 / 0)$ & $5(2 / 40)$ & $10(2 / 20)$ & $0(0 / 20)$ \\
\hline Exacerbation COPD & $25(5 / 20)$ & $30(3 / 10)$ & $20(2 / 10)$ & $12.5(5 / 40)$ & $15(3 / 20)$ & $10(2 / 20)$ \\
\hline Neurologic dysfunction & $10(2 / 20)$ & $10(1 / 10)$ & $10(1 / 10)$ & $32.5(13 / 40)$ & $40(8 / 20)$ & $25(5 / 20)$ \\
\hline Sepsis & $0(0 / 0)$ & $0(0 / 0)$ & $0(0 / 0)$ & $12.5(5 / 40)$ & $5(1 / 20)$ & $20(4 / 20)$ \\
\hline Other & $5(1 / 20)$ & $10(1 / 10)$ & $0(0 / 0)$ & $2.5(2 / 40)$ & $5(1 / 20)$ & $5(1 / 20)$ \\
\hline Successfully extubated during study, \% (n/N) & $60(12 / 20)$ & $80(8 / 10)$ & $40(4 / 10)$ & $65(26 / 40)$ & $80(16 / 20)$ & $50(10 / 20)$ \\
\hline
\end{tabular}

Values are presented as mean \pm standard deviation, or as absolute or percentage number of patients

COPD chronic obstructive pulmonary disease, $\mathrm{FiO}_{2}$ fraction of inspired oxygen, $I C U$ intensive care unit, $M V$ mechanical ventilation

patients) and were reasons for an early stop of the current expiratory muscle FES session (Table 2). Device-related adverse events reported in the sham group included hypertension and tachycardia ( $n=3$, in one patient). No FES-related serious adverse events were reported, as judged by a physician and confirmed by the local ethics board.

For the total population, the threshold intensity for strong muscle contraction was 70 [54-73] $\mathrm{mA}$ and the maximum tolerated intensity was 100 [60-100] mA, as assessed prior to randomization. Muscle contraction was confirmed during all active sessions. The average stimulation intensity remained stable over the study period (change of $-0.9[-2.1-0.0] \%$ ). The patient's average session-to-session change in stimulation intensity (either an increase or decrease as compared to the previous session) was 4.8 [3.0-7.1] \%. Additional file 1: Figure 3 shows that with higher Richmond Agitation-Sedation Scale, the applied stimulation intensity was lower compared to the patient's average intensity $(P=0.02)$. Most active sessions were performed during pressure support ventilation (PSV) (percentage of sessions during PSV: $96.4[59.6-100] \%)$.

\section{Inflammatory markers}

Figure 2a presents the plasma cytokine levels at baseline and on day $3(N=$ active/sham; 9/9). There was a between-group difference in the change in pro-inflammatory marker TNF- $\alpha(P=0.03)$, with a trend toward a decrease in TNF- $\alpha$ levels for the active group versus no change in the sham group. Mean differences compared to baseline were $-0.15 \pm 0.20 \mathrm{pg} / \mathrm{mL}(P=0.05)$ versus $0.02 \pm 0.06 \mathrm{pg} / \mathrm{mL}(P=0.32)$, respectively.

\section{Pooled analysis}

See Table 1 for characteristics of the pooled study population $(N=$ active/sham: $20 / 20)$.

\section{Ultrasound}

Due to technical issues, ultrasound data of four Holland study patients from one site were incomplete (two active and two sham patients). Missing data for the Australian study are addressed in [17]. In patients with adequate baseline measurements available and who completed $\geq 75 \%$ of stimulation sessions from the intend-totreat set, total abdominal expiratory muscle thickness at baseline was 11.7 [10.6-20.6] versus 12.4 [10.5-15.5] $\mathrm{mm}$ for the active $(N=9)$ versus sham $(N=17)$ group, respectively. There was a between-group difference in the change in total abdominal expiratory muscle thickness on day 3; mean (95\% confidence interval (CI)) changes from baseline on day 3 were $1.76(0.21,3.30)$ versus $-0.50(-1.56,0.57) \mathrm{mm}$ for the active $(N=7)$ and sham $(N=12)$ group, respectively, with a treatment difference $(95 \% \mathrm{CI})$ of $2.25(0.34,4.16) \mathrm{mm}(P=0.02)$ (Fig. 3). No between-group difference in total abdominal expiratory muscle thickness was found on day 5 . Ultrasound results for all individual respiratory muscles are presented in Additional file 1: Tables 1-6; no changes between groups or over days were found. 
Table 2 Compliance to expiratory muscle functional electrical stimulation (FES) sessions and reported adverse events (AE) during the study period

\begin{tabular}{|c|c|c|c|c|c|c|}
\hline Treatment compliance & \multicolumn{2}{|c|}{ Sham $(N=10)$} & \multicolumn{3}{|c|}{ Active $(N=10)$} & Total $(N=20)$ \\
\hline Total sessions, $n$ & \multicolumn{2}{|c|}{103} & \multicolumn{2}{|c|}{169} & & 272 \\
\hline 30-min sessions completed, $n$ ( $\%$ of total sessions) & \multicolumn{2}{|c|}{$98(95.1)$} & \multicolumn{2}{|c|}{$147(87)$} & & $245(91.1)$ \\
\hline \multicolumn{7}{|l|}{ Reasons for early stop of current sessiona,$\%(n / N)$} \\
\hline Heart rate $<40 \mathrm{bpm}$ & \multicolumn{2}{|c|}{$0(0 / 103)$} & \multicolumn{2}{|c|}{$0(0 / 169)$} & & $0(0 / 272)$ \\
\hline Heart rate $>130 \mathrm{bpm}$ & \multicolumn{2}{|c|}{$1(1 / 103)$} & \multicolumn{2}{|c|}{$0.6(1 / 169)$} & & $0.7(2 / 272)$ \\
\hline $\mathrm{MAP}<60 \mathrm{mmHg}$ & \multicolumn{2}{|c|}{$0(0 / 103)$} & \multicolumn{2}{|c|}{$0(0 / 169)$} & & $0(0 / 272)$ \\
\hline MAP > $110 \mathrm{mmHg}$ & \multicolumn{2}{|c|}{$1.9(2 / 103)$} & \multicolumn{2}{|c|}{$6.5^{*}(11 / 169)$} & & $4.8(13 / 272)$ \\
\hline Respiratory rate $>40$ breaths/min & \multicolumn{2}{|c|}{$1(1 / 103)$} & \multicolumn{2}{|c|}{$3(5 / 169)$} & & $2.2(6 / 272)$ \\
\hline $\mathrm{SpO}_{2}<90 \%$ & \multicolumn{2}{|c|}{$1(1 / 103)$} & \multicolumn{2}{|c|}{$0(0 / 169)$} & & $0.4(1 / 272)$ \\
\hline Behavior pain scale $>4$ & \multicolumn{2}{|c|}{$0(0 / 103)$} & \multicolumn{2}{|c|}{$0.6(1 / 169)$} & & $0.4(1 / 272)$ \\
\hline Patient request to stop & \multicolumn{2}{|c|}{$0(0 / 103)$} & \multicolumn{2}{|c|}{$2.4(4 / 169)$} & & $1.5(4 / 272)$ \\
\hline Treatment days from intent-to-treat set, $\%$ (median $\left(q_{1}-q_{3}\right)$ ) & \multicolumn{2}{|c|}{$100(69-100)$} & \multicolumn{2}{|c|}{$89(50-100)$} & & $100(50-100)$ \\
\hline \multicolumn{7}{|l|}{ Reasons for treatment stop before extubation, patients \% $(n / N)$} \\
\hline Discomfort & \multicolumn{2}{|c|}{$10(1 / 10)$} & \multicolumn{2}{|c|}{$10(1 / 10)$} & & $10(2 / 20)$ \\
\hline Switch to NAVA mode ${ }^{b}$ & \multicolumn{2}{|c|}{$10(1 / 10)$} & \multicolumn{2}{|c|}{$20(2 / 10)$} & & $15(3 / 20)$ \\
\hline Patient request to stop & \multicolumn{2}{|c|}{$0(0 / 10)$} & \multicolumn{2}{|c|}{$10(1 / 10)$} & & $5(1 / 20)$ \\
\hline Safetyc & Event & $\begin{array}{l}\text { Patient } \\
\%(n / N)\end{array}$ & Event & $\begin{array}{l}\text { Patient } \\
\%(n / N)\end{array}$ & Event & $\begin{array}{l}\text { Patient } \\
\%(n / N)\end{array}$ \\
\hline Adverse events (any) & 11 & $80(8 / 10)$ & 17 & $60(6 / 10)$ & 28 & $70(14 / 20)$ \\
\hline Unanticipated adverse device effects & 0 & $0(0 / 10)$ & 9 & $40(4 / 10)$ & 9 & $20(4 / 20)$ \\
\hline AEs leading to discontinuation of current FES session ${ }^{d}$ or study & 8 & $60(6 / 10)$ & 14 & $50(5 / 10)$ & 22 & $55(11 / 20)$ \\
\hline Serious AEs - total & 7 & $60(6 / 10)$ & 3 & $20(2 / 10)$ & 10 & $40(8 / 20)$ \\
\hline Serious AEs_-deaths & 5 & $50(5 / 10)$ & 1 & $10(1 / 10)$ & 6 & $30(6 / 20)$ \\
\hline Intervention-related adverse events ${ }^{e}$ & 3 & $10(1 / 10)$ & 13 & $50(5 / 10)$ & 16 & $30(6 / 20)$ \\
\hline Unanticipated adverse device effects & 0 & $0(0 / 10)$ & 9 & $40(4 / 10)$ & 9 & $20(4 / 20)$ \\
\hline AEs leading to discontinuation of current FES session ${ }^{d}$ or study & 3 & $10(1 / 10)$ & 11 & $50(5 / 10)$ & 14 & $30(6 / 20)$ \\
\hline Serious AEs - total & 0 & $0(0 / 10)$ & 0 & $0(0 / 10)$ & 0 & $0(0 / 20)$ \\
\hline Serious AEs — deaths & 0 & $0(0 / 10)$ & 0 & $0(0 / 10)$ & 0 & $0(0 / 20)$ \\
\hline
\end{tabular}

MAP mean arterial pressure, $\mathrm{SpO}_{2}$ oxygen saturation, NAVA neurally adjusted ventilatory assist

* Six of these episodes developed in one patient who already had a high MAP prior to the FES session

a Also counted as adverse event, see safety parameters

b Stimulation artifacts were visible in the diaphragm electrical activity (EAdi) signal, limiting the application of NAVA mode. As the study protocol did not allow to change ventilator mode, expiratory muscle FES sessions were discontinued in patients ventilated in NAVA.

c Participants that experienced multiple adverse events within a specific category are counted only once in the calculation of occurrence rate for that category

d Discontinuation of current expiratory muscle FES session based on stop criteria.

e Intervention-related adverse events include definitely, probably and possibly relationships

\section{Inflammatory markers}

Figure $2 \mathrm{~b}$ presents the results for plasma cytokine levels at baseline and on day 3 ( $N=$ active/sham; $19 / 17)$; no differences between groups or over days were found.

\section{Clinical outcomes}

Figure 4 shows results for extubation success and ICU length of stay. Using the cumulative incidence competing risks method for the total study population, median ventilation duration and ICU length of stay were 10 versus 52 days $(P=0.07)$, and 12 versus 54 days $(P=0.03)$ for the active versus sham group, respectively. For patients that were successfully extubated during ICU stay, the median ventilation duration was $8.5[5.6-12.2]$ versus $10.5[5.3-25.6]$ days $(P=0.60)$ for the active $(N=16)$ versus sham $(N=10)$ group, respectively. Median ICU length of stay was 10.5 [8.0-14.5] versus 14.0 [9.0-19.5] days $(P=0.36)$ for those active $(N=16)$ versus sham $(N=8)$ patients that were extubated and discharged alive from the ICU. During the 

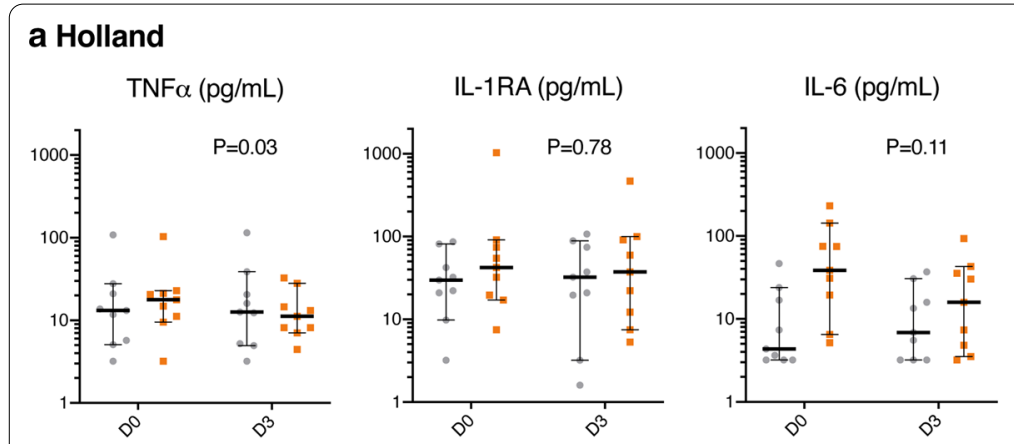

IL-8 (pg/mL)

IL-10 (pg/mL)

\section{b Pooled}
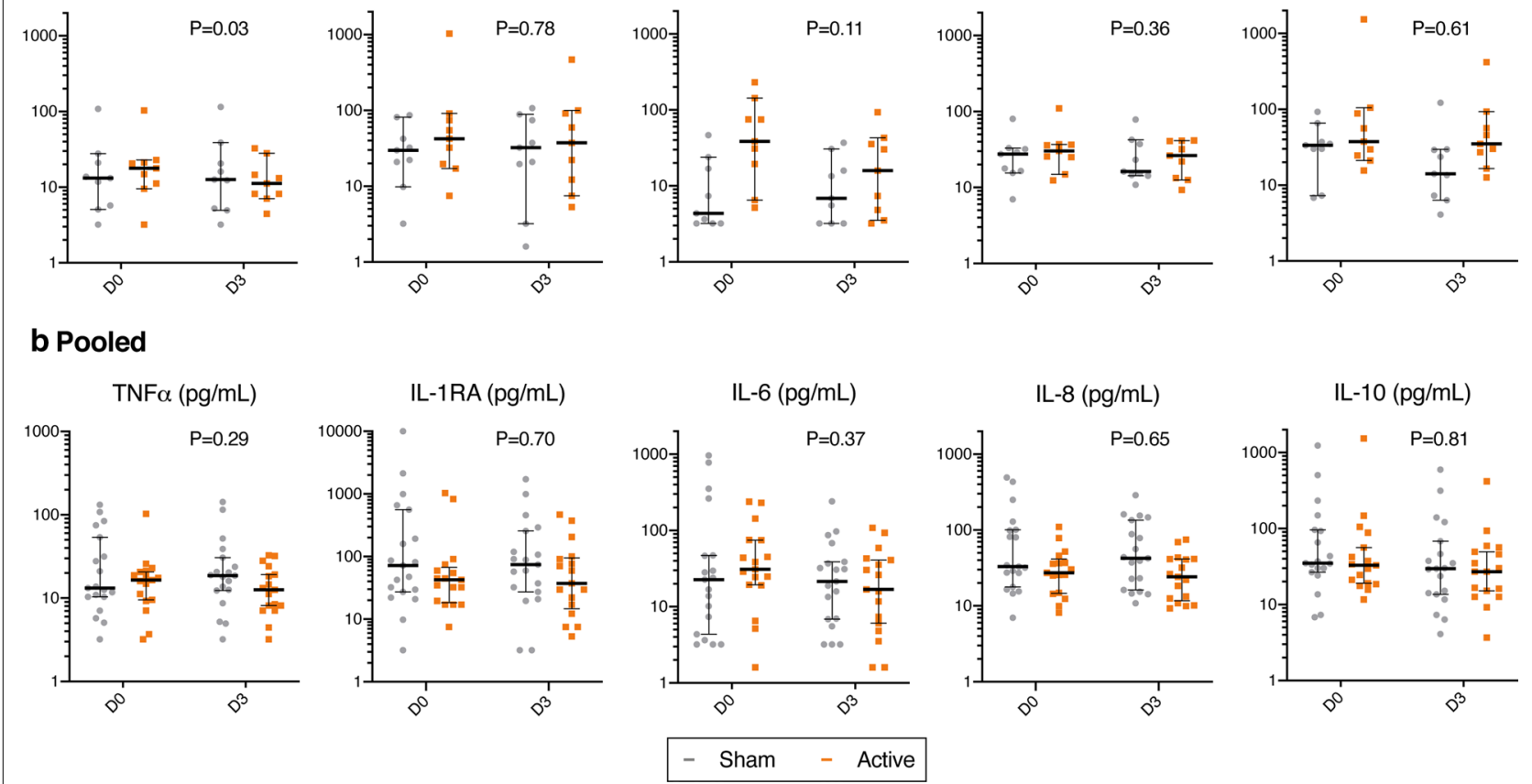

Fig. 2 Systemic inflammatory markers obtained at study enrollment (baseline, D0) and on the third treatment day (D3). a Holland study ( $N=$ active/ sham; 9/9), b pooled analysis ( $N=17 / 19)$. Measured cytokines included tumor necrosis factor (TNF)-a, interleukin (IL)-1 receptor antagonist (RA), $I L-6, I L-8$, and IL-10. Levels of IL-1 $\beta$ were for $96 \%$ of the samples below the limit of quantitation and are therefore not presented. Data are presented as medians with interquartile ranges. Due to the large between-subject variability in inflammatory markers, data are presented on a logarithmic scale. Log-transformed data were analyzed using a two-way analysis of variance with factors of time (D0, D3) and group. $P$ values represent the interaction effect of group $\times$ time

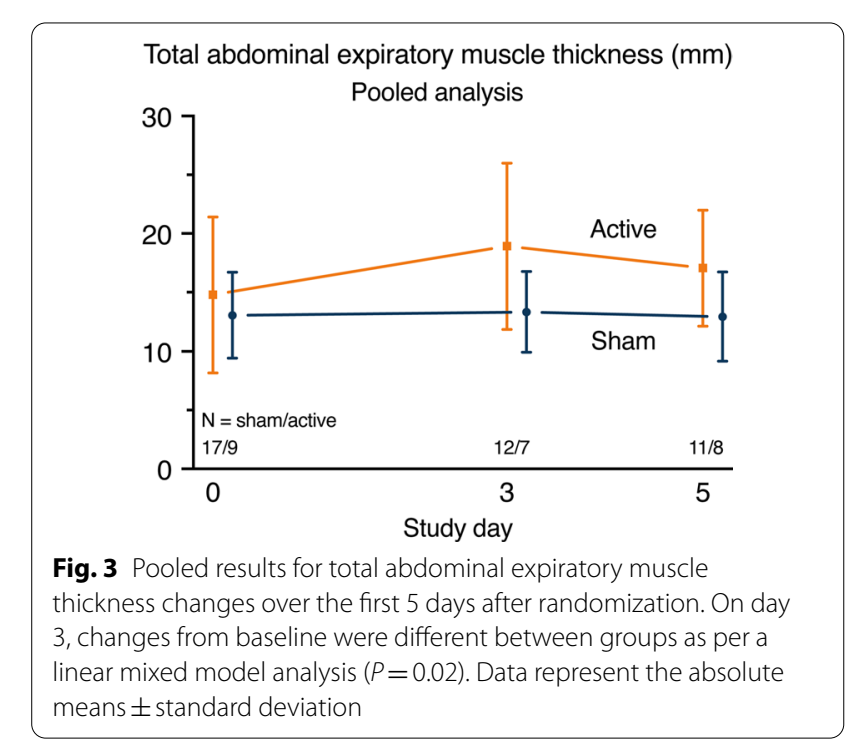

ICU stay, 3/20 (15\%) patients died in the active group, versus $8 / 20(40 \%)$ patients in the sham group $(P=0.16)$.

\section{Discussion}

The current feasibility study demonstrates that breathsynchronized expiratory muscle FES is feasible, safe and effective in eliciting expiratory muscle activity during mechanical ventilation in ICU patients. This supports the findings of the Australian study [17], but in a more heterogeneous ICU population. The pooled analysis of the Australian and Holland studies also provides important insights for the design of future studies to evaluate whether this approach could improve weaning and ventilator liberation outcome.

\section{Rationale for expiratory muscle FES}

Recent studies suggest that maintaining diaphragm activity during mechanical ventilation minimizes diaphragm disuse atrophy and may improve clinical outcome $[4,20]$. The current study was designed to investigate the feasibility and efficacy of eliciting expiratory muscle activity in the early stages of mechanical ventilation, based on the 

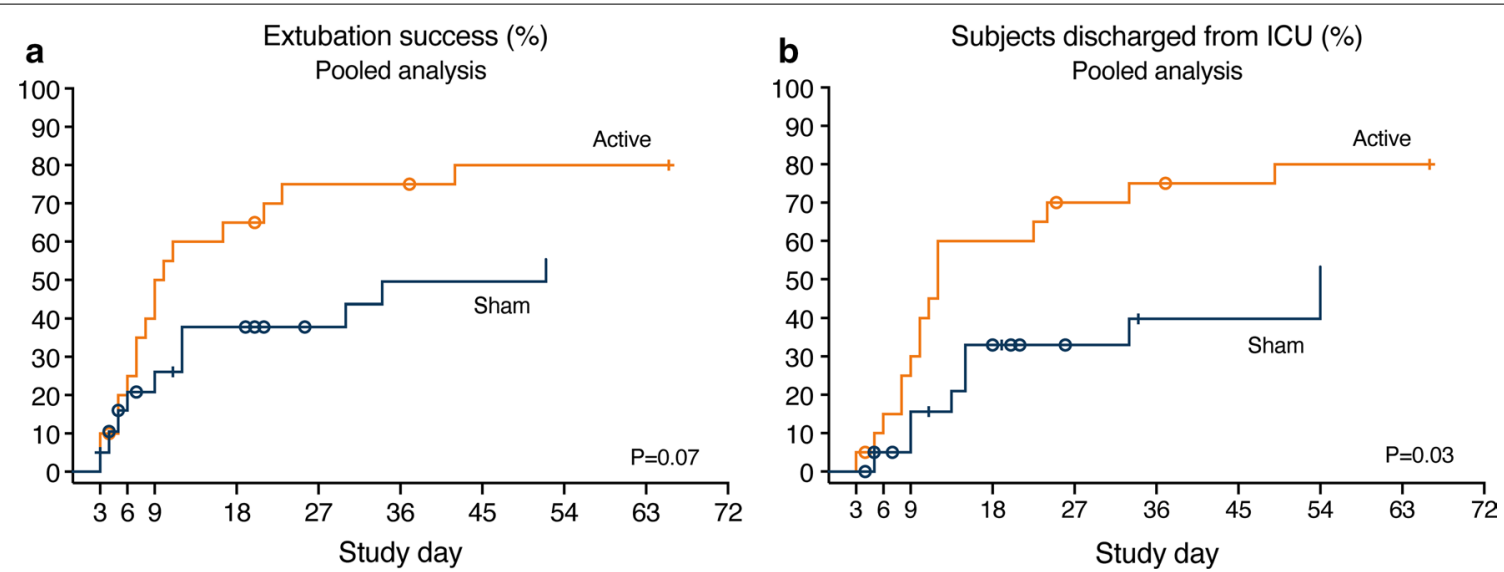

Fig. 4 Pooled results on clinical endpoints. a Pooled results on extubation success. b Pooled results on ICU length of stay. P values are based on Gray's test. Cumulative event rates were estimated based on competing risk analysis, with the competing risks of death or withdrawal of ICU treatment (e.g., ventilator support) with the intention of subsequent death. Symbols: o for competing events; + for censored data

assumption that mechanical ventilation is associated with expiratory muscle disuse atrophy. While this assumption has not been extensively studied, it is known that controlled mechanical ventilation may (partly) silence respiratory centers in the brainstem, resulting in disuse of the inspiratory and expiratory muscles [21]. In addition, data on rectus abdominis biopsies show that critically ill patients exhibit smaller myofiber cross-sectional area compared with controls [22]. The effects of critical illness and mechanical ventilation on the change in expiratory muscle thickness, however, are yet unknown but may be of clinical relevance as increasing evidence demonstrates expiratory muscle weakness at the time of ventilator weaning $[1,9,10,13,23]$, likely as a consequence of muscle disuse. Potential explanations for how expiratory muscle weakness affect weaning or extubation outcome include inadequate secretion clearance and insufficient cough capacity, resulting in respiratory complications such as pneumonia and atelectasis. Also, the expiratory muscles support inspiration in the presence of diaphragm dysfunction [1]; weakness may thus result in reduced ventilatory capacity. In line with our earlier results [17], we report more successful extubations for the pooled active group; however, this needs confirmation in an appropriately powered study.

\section{Feasibility, contractile response and safety}

We demonstrate that expiratory muscle FES as applied twice daily in the Holland study with a maximum intensity of $100 \mathrm{~mA}$ is feasible and safe in selected critically ill patients and allows high therapy compliance after an adequate contractile response to FES was verified. No FESrelated serious harm or complications were reported, and only few sessions were stopped early after meeting safety criteria.

Over the last decade, inconclusive evidence for the clinical benefits of FES in ICU patients was published $[15,24]$, mainly targeting limb muscles. One reason for these inconsistent results may be the lack of reporting of treatment compliance and contractile response to stimulation [25]. The effectiveness of FES in activating muscles at an adequate level depends on patient characteristics; factors such as sepsis, edema and vasopressor may play a role [26, 27]. No potential explanatory factors hampering contractile response were found in our patients that did not pass the expiratory muscle FES eligibility test $(N=2$ for maximum intensity of $60 \mathrm{~mA}$ (no data available on whether these patients would respond at higher intensities), $N=3$ for maximum intensity of $100 \mathrm{~mA}$ ). Besides changes in Richmond Agitation-Sedation Scale, no clinical or ventilator parameters were associated with changes in applied stimulation intensity for the active group. The latter could be explained by the fact that stimulation intensity remained relatively stable, while ICU patients show more day-to-day variation in clinical signs.

Interestingly, our high success rate of contractile activation is not in line with Grunow et al. [25], studying contractile response to FES (verified visually or on palpation) in eight limb muscle groups and reporting that only $64.4 \%$ of applied stimulations led to an adequate response on the day of ICU admission; this number declined to $25 \%$ after one week. Although the expiratory muscles were not targeted in their study, their maximum stimulation intensity was $70 \mathrm{~mA}$, i.e., our median threshold intensity. While a strong muscle contraction depends on many factors (e.g., pulse duration, skin/electrode interface, electrode size and location to motor point), it is 
plausible that increasing intensity would provide higher contractile response rates in their study. In addition, we used ultrasound to verify the initial response, which we consider a more objective measure compared to palpation, especially in patients with high body mass index or edema. Lastly, we did not experience decreases in contractile response throughout the study, likely due to the higher intensities used and pre-randomization stimulation titration.

Plasma cytokine levels were measured to evaluate whether early application of FES was associated with enhanced systemic inflammatory response. Starting expiratory muscle FES as early as possible is important, as respiratory muscle atrophy largely develops within the first 4 days of mechanical ventilation [20,29]. However, it is important to assess whether patients tolerate the increased physical demands and whether early FES causes an inflammatory state. We did not find associations with systemic inflammatory response, but with the large variability in pro-inflammatory state of ICU patients, no clear implications can be generated about potential protective effects. This is in line with recent work [30] on limb muscle FES in ICU patients and with data in healthy subjects demonstrating that the effects of FES are similar to those of mild exercise [31], i.e., inhibiting pro-inflammatory cytokines [32]. Furthermore, Hickmann et al. [33] showed that early exercise during the onset of septic shock did not enhance inflammation and preserved muscle mass. Similar mechanisms may explain potential protective effects of FES on muscle loss, but this requires repeated measurements of cytokines during the full period of a FES protocol, which was not the focus of our study and would be of interest to address in future research.

There are yet no clinical applications of FES targeting the respiratory muscles of ICU patients under mechanical ventilation. Transvenous phrenic nerve pacing is currently being studied as potential intervention for improving diaphragm strength in difficult-to-wean patients (NCT03096639). However, this technique is invasive, increasing risks associated with subclavian vein cannulation and blood stream infections. In contrast, we focused on employing breath-synchronized expiratory muscle FES in the early phase of critical illness, aiming to prevent (or attenuate) the development of muscle disuse in selected patients with an anticipated expected prolonged duration of mechanical ventilation. We reason that this could be a novel noninvasive application within a respiratory muscle-protective ventilation strategy.

\section{Potential effects on clinical endpoints}

For the pooled data, we observed a between-group difference in total abdominal expiratory muscle thickness changes on day 3, favoring the active group. Although effects were small, our observations are in line with Dall'Acqua et al. [28], showing that FES of the rectus abdominis muscle (note that this muscle is to a limited extent involved in expiration [1], therefore not specifically targeted in our study) of ICU patients resulted in muscle mass preservation in the active group, while thickness decreased in the sham group. However, we found no between-group differences on day 5, likely resulting from insufficient sample size and observer variability (see 'Strengths and limitations' section).

In the pooled analysis of clinical outcomes using the cumulative incidence competing risks method, we report between-group differences in median ventilation duration and ICU length of stay. As the incidence rate of the event of interest was influenced by competing events (particularly death in the sham group) and the estimates might not be stable given the small sample size, we also calculated the median ventilation duration and ICU length of stay for those patients that experienced the event of interest. No between-group differences were found for these subgroups. A next step would be to test the treatment effect of expiratory muscle FES in a study powered on clinical endpoints. Assuming a median effect size with $60 \%$ of patients successfully extubated on day 9 for the intervention group versus $45 \%$ of patients in the sham group, a next study would require 254 participants (hazard approach; two-sided log-rank test with two-sided alpha of 0.05 , beta of 0.1 , mortality on day 9 of $20 \%$, and $10 \%$ of patients lost to follow-up).

\section{Strengths and limitations}

Strengths are that we enrolled a heterogeneous group of patients from different centers and performed a relatively high number $(n=272)$ of FES sessions. Also, contractile response was verified prior to randomization, resulting in high treatment compliance and to ensure that neuromuscular status was comparable between groups. Lastly, a pooled analysis was performed, including evaluation of cytokines, to assess potential benefits in a larger cohort.

This study has some limitations. First, it was designed with the assumption that ultrasound could provide sufficient insights into the effects of expiratory muscle FES on muscle mass preservation. Despite using a standardized protocol [19], obtaining reliable ultrasound measurements in ICU patients can be challenging. Changes in expiratory muscle thickness can reflect actual changes in muscle thickness (i.e., atrophy or hypertrophy), but could also be affected by observer variability and patient characteristics. For example, motion of abdominal contents with respiration could passively stretch the abdominal expiratory muscles. 
Also, abdominal expiratory muscles have more degrees of freedom to move compared to the diaphragm; active contraction of one muscle layer could directly influence the position of the adjacent layer. For this reason, we evaluated changes in total abdominal expiratory muscle thickness and used a linear mixed model to account for individual changes, but the study was not powered sufficiently to draw any conclusions on these results. In contrast, ultrasound is valuable for verifying contractile response to stimulation (Additional file 1: Figure 4). Second, sample size was small and patients were enrolled relatively late after intubation. This resulted in a highly selected study population prone to prolonged mechanical ventilation. Because of this reason, the absence of protocolized ventilator and weaning strategies, and post-randomization events (particularly death in the sham group), results on clinical outcomes should be interpreted with caution and generalizability of the findings is limited. Third, this study lacks a robust outcome parameter to assess physiological effects of expiratory muscle FES, including dose-response relationships. The dosage of 30-min stimulation sessions twice daily was chosen based on a few practical and physiological considerations. First, while the rate of expiratory muscle atrophy is yet unknown, diaphragm atrophy rapidly occurs after the start of mechanical ventilation $[20,29]$. Hence, we wanted to limit the delay between stimulation sessions to a maximum duration of $24 \mathrm{~h}$, which is only possible to guarantee by including more than one stimulation session per day. Second, it is known that the force evoked from a muscle by electrical stimulation could decline rapidly over time because of repetitive activation of the same motor units. We therefore reasoned that limiting the stimulation session duration to $30 \mathrm{~min}$ would help to ensure a strong muscle contraction throughout the session. Other considerations included availability of study personnel and possible interference with clinical protocols/activities. Nevertheless, a next study should consider different FES protocols in order to find the optimal session frequency and duration for improving clinical outcomes. Moreover, although it would be interesting to assess muscle changes on a cellular or functional level and in response to different expiratory muscle FES protocols, such measurements would require repeated muscle biopsies or assessment of gastric twitch pressures in response to stimulation of the expiratory muscle nerve roots, respectively. We did not perform these invasive and technically challenging techniques in our study, but focused on the feasibility and efficacy of employing expiratory muscle FES in the early phase of ICU stay. Addressing such physiological endpoints would be of interest in order to better understand the potential effects of expiratory muscle FES on maintaining muscle function.

\section{Conclusion}

Breath-synchronized expiratory muscle FES is a feasible and generally safe intervention to elicit expiratory muscle activity during the early stages of mechanical ventilation in selected ICU patients. This could be a novel intervention within a respiratory muscle-protective ventilation strategy. The effects of expiratory muscle FES on weaning and ventilator liberation outcome remain to be studied.

\section{Supplementary information}

Supplementary information accompanies this paper at https://doi. org/10.1186/s13054-020-03352-0.

Additional file 1. Extended methods and results.

Abbreviations

FES: Functional electrical stimulation; ICU: Intensive care unit; IL: Interleukin; PSV: Pressure support ventilation; TNF: Tumor necrosis factor.

\section{Acknowledgements}

The authors would like to thank John Shen and Jason Cai from OcTech Medical for their statistical contribution to the project and the final manuscript.

\section{Authors' contributions}

AHJ, AJM, EO, PHV, and LMAH developed the Holland study design and protocol. AHJ, TF, OH, MK, MP, PP, LHR, JS, ZS, HJdV, CW, YZ, and LMAH performed data collection for the Holland study. EJM, CLBR, DWC, SCG, JEB were part of the Australian study team (see [17] for full contributions); EJM, DWC, JEB, and CSW performed additional data collection for the Australian study. AHJ and LMAH performed data analysis of the Holland and pooled study. All authors were involved in data interpretation. The first draft was written by AHJ and $L M A H$. All authors commented on previous versions of the manuscript. All authors read and approved the final manuscript.

\section{Funding}

This study was an investigator-initiated study. It was funded by the participating hospitals and supported by a research grant from Liberate Medical. AJM is a Liberate Medical employee. He assisted in the study design and reviewed the manuscript, without modifying the content or conclusions. Liberate Medical had no role in the collection of data.

\section{Availability of data and materials}

The datasets and protocols used and analyzed during the current study are available from the corresponding author on reasonable request.

\section{Ethics approval and consent to participate}

The study was approved by the ethics board of the Radboud University Medical Center (METC 2016-2366) and locally approved by participating centers (Amsterdam UMC, location VUmc: METC 2016.514; Canisius Wilhelmina Ziekenhuis: METC 128-2017). As patients were lacking the capacity to provide consent, written informed consent was provided by the substitute decisionmaker prior to study procedures.

\section{Consent for publication}

Not applicable.

\section{Competing interests}

AJM is employed by Liberate Medical, a medical device company that is developing an expiratory muscle stimulator. AHJ, TF, LMAH have received personal 
fees from Liberate Medical, outside of the submitted work. The other authors do not declare competing interests.

\section{Author details}

${ }^{1}$ Department of Intensive Care Medicine, Amsterdam University Medical Centers, location VUmc, Postbox 7505, 1007 MB Amsterdam, The Netherlands. ${ }^{2}$ Amsterdam Cardiovascular Sciences Research Institute, Amsterdam UMC, Amsterdam, The Netherlands. ${ }^{3}$ Department of Intensive Care Medicine, Radboud University Medical Center, Nijmegen, The Netherlands. ${ }^{4}$ Neuroscience Research Australia, 139 Barker Street, Randwick, NSW 2031, Australia. 5 School of Medical Sciences, University of New South Wales, Kensington, NSW 2052, Australia. ${ }^{6}$ Liberate Medical LLC, Crestwood, KY 40014, USA. ${ }^{7}$ Prince of Wales Hospital, Randwick, NSW 2031, Australia. ${ }^{8}$ Department of Intensive Care Medicine, Canisius Wilhelmina Hospital, Nijmegen, The Netherlands. ${ }^{9}$ Cardiovascular and Respiratory Physiology Group, Technical Medical Centre, University of Twente, Enschede, The Netherlands. ${ }^{10}$ Department of Biomedical Signals and Systems, Technical Medical Centre, University of Twente, Enschede, The Netherlands. ${ }^{11}$ School of Psychiatry, University of New South Wales, Kensington, NSW 2052, Australia. ${ }^{12}$ Department of Neuroscience and Physiology, Upstate Medical University, New York 13210, USA.

Received: 27 July 2020 Accepted: 16 October 2020

Published online: 30 October 2020

\section{References}

1. Shi ZH, Jonkman A, de Vries H, Jansen D, Ottenheijm C, Girbes A, et al. Expiratory muscle dysfunction in critically ill patients: towards improved understanding. Intensive Care Med. 2019;45(8):1061-71.

2. De Troyer A, Boriek AM. Mechanics of the respiratory muscles. Compr Physiol. 2011;1(3):1273-300.

3. Dres M, Goligher EC, Heunks LMA, Brochard LJ. Critical illness-associated diaphragm weakness. Intensive Care Med. 2017;43(10):1441-52.

4. Goligher EC, Dres M, Fan E, Rubenfeld GD, Scales DC, Herridge MS, et al. Mechanical ventilation-induced diaphragm atrophy strongly impacts clinical outcomes. Am J Respir Crit Care Med. 2018;197(2):204-13.

5. Dres M, Dube BP, Mayaux J, Delemazure J, Reuter D, Brochard L, et al. Coexistence and impact of limb muscle and diaphragm weakness at time of liberation from mechanical ventilation in medical intensive care unit patients. Am J Respir Crit Care Med. 2017;195(1):57-66.

6. Dres M, Jung B, Molinari N, Manna F, Dube BP, Chanques G, et al. Respective contribution of intensive care unit-acquired limb muscle and severe diaphragm weakness on weaning outcome and mortality: a post hoc analysis of two cohorts. Crit Care. 2019;23(1):370.

7. Adler D, Dupuis-Lozeron E, Richard J-C, Brochard L. Does inspiratory muscle dysfunction predict readmission after intensive care unit discharge? Am J Respir Crit Care Med. 2014;190(3):347-9.

8. Savi A, Teixeira C, Silva JM, Borges LG, Pereira PA, Pinto KB, et al. Weaning predictors do not predict extubation failure in simple-to-wean patients. J Crit Care. 2012;27(2):221 e1-8.

9. Su WL, Chen YH, Chen CW, Yang SH, Su CL, Perng WC, et al. Involuntary cough strength and extubation outcomes for patients in an ICU. Chest. 2010;137(4):777-82.

10. Epstein SK. Decision to extubate. Intensive Care Med. 2002;28(5):535-46

11. Ottenheijm C, Heunks L. Diaphragm-protective mechanical ventilation to improve outcomes in ICU patients? Am J Respir Crit Care Med. 2018;197(2):150-2.

12. Reynolds SC, Meyyappan R, Thakkar V, Tran BD, Nolette MA, Sadarangani $G$, et al. Mitigation of ventilator-induced diaphragm atrophy by transvenous phrenic nerve stimulation. Am J Respir Crit Care Med. 2017;195(3):339-48.

13. Salam A, Tilluckdharry L, Amoateng-Adjepong Y, Manthous CA. Neurologic status, cough, secretions and extubation outcomes. Intensive Care Med. 2004;30(7):1334-9.

14. De Jonghe B, Bastuji-Garin S, Durand MC, Malissin I, Rodrigues P, Cerf C, et al. Respiratory weakness is associated with limb weakness and delayed weaning in critical illness. Crit Care Med. 2007;35(9):2007-15.

15. Williams N, Flynn M. A review of the efficacy of neuromuscular electrical stimulation in critically ill patients. Physiother Theory Pract. 2014;30(1):6-11.
16. Maffiuletti NA, Roig M, Karatzanos E, Nanas S. Neuromuscular electrical stimulation for preventing skeletal-muscle weakness and wasting in critically ill patients: a systematic review. BMC Med. 2013;11:137.

17. McCaughey EJ, Jonkman AH, Boswell-Ruys CL, McBain RA, Bye EA, Hudson AL, et al. Abdominal functional electrical stimulation to assist ventilator weaning in critical illness: a double-blinded, randomised, sham-controlled pilot study. Crit Care. 2019;23(1):261.

18. McCaughey EJ, McLachlan AJ, Gollee H. Non-intrusive real-time breathing pattern detection and classification for automatic abdominal functional electrical stimulation. Med Eng Phys. 2014;36(8):1057-61.

19. Tuinman PR, Jonkman AH, Dres M, Shi ZH, Goligher EC, Goffi A, et al. Respiratory muscle ultrasonography: methodology, basic and advanced principles and clinical applications in ICU and ED patients - a narrative review. Intensive Care Med. 2020;46:594-605.

20. Goligher EC, Fan E, Herridge MS, Murray A, Vorona S, Brace D, et al. Evolution of diaphragm thickness during mechanical ventilation. Impact of inspiratory effort. Am J Respir Crit Care Med. 2015;192(9):1080-8.

21. Guyenet PG, Stornetta RL, Souza G, Abbott SBG, Shi Y, Bayliss DA. The retrotrapezoid nucleus: central chemoreceptor and regulator of breathing automaticity. Trends Neurosci. 2019;42(11):807-24.

22. Derde S, Hermans G, Derese I, Guiza F, Hedstrom Y, Wouters PJ, et al. Muscle atrophy and preferential loss of myosin in prolonged critically ill patients. Crit Care Med. 2012;40(1):79-89.

23. McCool FD. Global physiology and pathophysiology of cough: ACCP evidence-based clinical practice guidelines. Chest. 2006;129:48S-53S.

24. Kho ME, Truong AD, Zanni JM, Ciesla ND, Brower RG, Palmer JB, et al. Neuromuscular electrical stimulation in mechanically ventilated patients: a randomized, sham-controlled pilot trial with blinded outcome assessment. J Crit Care. 2015;30(1):32-9.

25. Grunow JJ, Goll M, Carbon NM, Liebl ME, Weber-Carstens S, Wollersheim T. Differential contractile response of critically ill patients to neuromuscular electrical stimulation. Crit Care. 2019;23(1):308.

26. Segers J, Hermans G, Bruyninckx F, Meyfroidt G, Langer D, Gosselink R. Feasibility of neuromuscular electrical stimulation in critically ill patients. $J$ Crit Care. 2014;29(6):1082-8.

27. Poulsen JB, Moller K, Jensen CV, Weisdorf S, Kehlet H, Perner A. Effect of transcutaneous electrical muscle stimulation on muscle volume in patients with septic shock. Crit Care Med. 2011;39(3):456-61.

28. Dall'Acqua AM, Sachetti A, Santos LJ, Lemos FA, Bianchi T, Naue WS, et al. Use of neuromuscular electrical stimulation to preserve the thickness of abdominal and chest muscles of critically ill patients: a randomized clinical trial. J Rehabil Med. 2017;49(1):40-8.

29. Jaber S, Petrof BJ, Jung B, Chanques G, Berthet JP, Rabuel C, et al. Rapidly progressive diaphragmatic weakness and injury during mechanical ventilation in humans. Am J Respir Crit Care Med. 2011;183(3):364-71.

30. Silva PE, de Cassia MR, Livino-de-Carvalho K, de Araujo AET, Castro J, da Silva VM, et al. Neuromuscular electrical stimulation in critically ill traumatic brain injury patients attenuates muscle atrophy, neurophysiological disorders, and weakness: a randomized controlled trial. J Intensive Care. 2019;7:59.

31. Truong AD, Kho ME, Brower RG, Feldman DR, Colantuoni E, Needham DM. Effects of neuromuscular electrical stimulation on cytokines in peripheral blood for healthy participants: a prospective, single-blinded Study. Clin Physiol Funct Imaging. 2017;37(3):255-62.

32. Santos RV, Viana VA, Boscolo RA, Marques VG, Santana MG, Lira FS, et al. Moderate exercise training modulates cytokine profile and sleep in elderly people. Cytokine. 2012;60(3):731-5.

33. Hickmann CE, Castanares-Zapatero D, Deldicque L, van den Berg P, Caty $G$, Robert A, et al. Impact of very early physical therapy during septic shock on skeletal muscle: a randomized controlled trial. Crit Care Med. 2018;46(9):1436-43.

\section{Publisher's Note}

Springer Nature remains neutral with regard to jurisdictional claims in published maps and institutional affiliations. 